\title{
Testosterone and Adipokines are Determinants of Physical Performance, Strength, and Aerobic Fitness in Frail, Obese, Older Adults
}

\author{
Lina E. Aguirre, ${ }^{1,2}$ Irum Zeb Jan, ${ }^{1}$ Kenneth Fowler, ${ }^{1,2}$ Debra L. Waters, ${ }^{3,4}$ \\ Dennis T. Villareal, ${ }^{1,4}$ and Reina Armamento-Villareal ${ }^{1,4}$ \\ ${ }^{1}$ New Mexico VA Health Care System, 1501 San Pedro SE, Albuquerque, NM 87108, USA \\ ${ }^{2}$ Biomedical Research Institute of New Mexico, Albuquerque, NM 87108, USA \\ ${ }^{3}$ University of Otago, Dunedin 9016, New Zealand \\ ${ }^{4}$ University of New Mexico School of Medicine, Albuquerque, NM 87131, USA
}

Correspondence should be addressed to Lina E. Aguirre; aguirre.linal@gmail.com

Received 26 February 2014; Accepted 4 June 2014; Published 2 July 2014

Academic Editor: Nicola Napoli

Copyright (C) 2014 Lina E. Aguirre et al. This is an open access article distributed under the Creative Commons Attribution License, which permits unrestricted use, distribution, and reproduction in any medium, provided the original work is properly cited.

In this study, we evaluated the independent and combined effects of baseline circulating gonadal, anabolic hormones and adipokines on physical function in 107 frail, obese $\left(B M I \geq 30 \mathrm{~kg} / \mathrm{m}^{2}\right)$, and older ( $\geq 65 \mathrm{yr}$ ) subjects. Our results showed significant positive correlations between circulating testosterone and insulin growth factor-1 (IGF-1) with knee flexion, knee extension, one-repetition maximum (1-RM), and peak oxygen consumption $\left(\mathrm{VO}_{2}\right.$ peak), while no correlation was observed with estradiol. Among the adipokines, high sensitivity C-reactive protein (Hs-CRP) and leptin negatively correlated with the modified physical performance testing (PPT), knee flexion, knee extension, 1-RM, and $\mathrm{VO}_{2}$ peak. Interleukin-6 ( Il-6) negatively correlated with knee flexion and $\mathrm{VO}_{2}$ peak and soluble tumor necrosis factors receptor-1 (sTNFrl) correlated with PPT, 1-RM, and $\mathrm{VO}_{2}$ peak. Adiponectin correlated negatively with 1-RM. Multiple regression analysis revealed that, for PPT, sTNFrl was the only independent predictor. Independent predictors included adiponectin, leptin, and testosterone for knee flexion; leptin and testosterone for knee extension; adiponectin, leptin, and testosterone for 1-RM; and IGF-1, IL-6, leptin, and testosterone for $\mathrm{VO}_{2}$ peak. In conclusion, in frail obese older adults, circulating levels of testosterone, adiponectin, and leptin appear to be important predictors of physical strength and fitness, while inflammation appears to be a major determinant of physical frailty.

\section{Introduction}

A high body mass index (BMI) is associated with impairment in activities of daily living, limitations in mobility, decreased physical performance, and increased risk for functional decline [1] leading to increased nursing home admissions [2]. The elderly obese are especially susceptible to the adverse effects of excess body fat on physical function, because of (1) decreased muscle mass and strength which occur with aging and (2) the need to carry greater weight due to obesity [3]. Investigators from our group first reported that $96 \%$ of community-living older adults with BMIs $>30 \mathrm{~kg} / \mathrm{m}^{2}$ were frail, as determined by physical performance test scores, peak oxygen consumption $\left(\mathrm{VO}_{2}\right.$ peak), and ability to perform activities of daily living [3].

Aging is associated with the decline in gonadal and anabolic hormones and increased in inflammatory cytokines. These changes are associated with loss of muscle mass and decline in strength and physical function. An increase in lean body mass, strength, and physical function has been demonstrated with testosterone $[4,5]$ and growth hormone $(\mathrm{GH})[6]$ treatment among patients deficient of these hormones. On the other hand, aging is associated with increase in inflammatory cytokines, and high levels of these cytokines promote 
muscle catabolism and reduced muscle volume [7]. Since loss of muscle volume leads to reduced muscle strength and function, it is possible that the age-related hormonal decline and increased inflammation contribute to deterioration in physical function leading to frailty. Furthermore, in the obese elderly patient, the added increase in inflammatory cytokine production from the excess adipose tissues would enhance inflammation perhaps leading to worse frailty syndrome than nonobese elderly patients. However, little information is available on the contribution of the interaction of circulating hormones and adipokines (some of them proinflammatory) on frailty and physical disability in obese elderly adults.

The objective of this study was to evaluate the independent and combined effects of circulating gonadal (testosterone and estradiol) and anabolic hormones (growth hormone/IGF-1) and adipose-tissue derived factors (adipokines) on physical function, strength and physical fitness in frail obese older adults.

\section{Methods}

2.1. Study Design and Study Population. This study is a crosssectional analysis of baseline data from subjects who volunteered to participate in a previous lifestyle therapy trial of sedentary, frail, and elderly obese adults $[8,9]$. This study was conducted at Washington University School of Medicine in accordance with the guidelines in the Declaration of Helsinki for the ethical treatment of human subjects. The protocol was approved by the Washington University Institutional Review Board and a written informed consent was obtained from each subject. Participants were recruited through newspaper and radio advertisements. Inclusion/exclusion criteria were as reported previously $[8,9]$. Briefly, participants were $\geq 65$ years of age, with $\mathrm{BMI} \geq 30 \mathrm{~kg} / \mathrm{m}^{2}$, had sedentary lifestyle, (did not participate in regular exercise more than twice a week), had stable body weight $( \pm 2 \mathrm{~kg})$ over the past year, and were on stable medications for 6 months before enrollment. Participants who were treated with boneacting drugs (e.g., bisphosphonates, glucocorticoids, and sexsteroid compounds) during the previous year were excluded from participation. At enrollment, these subjects should not have cardiorespiratory or neuromuscular diseases that would limit their ability to exercise, diabetes mellitus, osteoporosis, hyperparathyroidism, chronic liver disease, uncontrolled or untreated hyperthyroidism, or significant renal impairment.

2.2. Physical Function. Physical function to determine frailty was assessed using the modified physical performance test (PPT) as previously described $[8,9]$. The modified PPT includes seven standardized tasks (walking $50 \mathrm{ft}$, putting on and removing a coat, picking up a penny, standing up from a chair, lifting a book, climbing one flight of stairs, and performing a progressive Romberg test) plus two additional tasks (climbing up and down four flights of stairs and performing a 360-degree turn). The score for each task ranges from 0 to 4; a perfect score is 36 .

\subsection{Muscle Strength Testing}

2.3.1. Knee Flexion and Knee Extension. Isokinetic knee extensor and flexor strength were evaluated by using a Biodex System 3 dynamometer (Shirley, NY) as previously described [10]. During the testing, the participants were seated with their backs supported and hips positioned at $120^{\circ}$ of flexion and secured to the seat of the dynamometer with thigh and pelvic straps. All tests were performed on the right leg. Testing was performed at an angular velocity of $60^{\circ}$ per second. The best of the 3 maximal voluntary efforts for each of the knee flexion and extension was used as the measure of absolute strength and reported as peak torque at $60^{\circ}$ in Newton-meter (N.m) units. The test-retest reliability of this method based on follow-up isokinetic testing done one week following the initial tests showed an intraclass correlation coefficient of 0.99 .

2.3.2. One Repetition Maximum. Total one-repetition maximum (1-RM) is the sum of the maximal weight a person can lift at one repetition for biceps curl, bench press, seated row, knee extension, knee flexion, and leg press $[8,10]$. The test-retest reliability of this method based on follow-up 1-RM determinations one week following the initial tests showed an intraclass correlation coefficient of 0.96 .

$\mathrm{VO}_{2}$ peak, a measure of aerobic fitness, was assessed during graded treadmill walking by indirect calorimetry (True Max 2400, ParvoMedics Salt Lake City, UT), as previously described [3,11]. Briefly, the incremental test started at a speed determined, during a warm-period, to elicit $\sim 70 \%$ of agepredicted $\mathrm{HR}_{\max }$, and the speed remained constant throughout the test, while the grade was increased by $2 \%$ every 2 minutes. The test continued until the subject could no longer exercise because of exhaustion or until other conditions, such as ECG changes or development of symptoms, made it unsafe to continue $[12,13]$.

2.3.3. Body Weight. Body weight was measured in the morning after the subjects had fasted for 12 hours [8]. BMI was calculated as weight in kilograms/square of the height in meters $\left(\mathrm{kg} / \mathrm{m}^{2}\right)$.

2.3.4. Biochemical Measurements. Blood samples were obtained in the morning after subjects fasted for at least 12 hours. Serum samples were extracted and stored at $-80^{\circ} \mathrm{C}$ until analysis. Enzyme-linked immunosorbent assay kits were used to measure interleukin-6 (IL-6) (Quantikine, R\&D Systems, Minneapolis, MN), soluble tumor necrosis factor receptor 1 (sTNFrl) (R\&D, Minneapolis, MN, USA), and adiponectin (Quantikine, R\&D Systems, Minneapolis, $\mathrm{MN}$ ). Radioimmunoassay kits were used to measure serum estradiol (Ultra-sensitive estradiol DSL-4800; Diagnostic Systems Laboratories Inc., Webster, Tex), leptin (Leptin HL-81K; Linco Research Inc., St Charles, MO), and insulinlike growth factor 1 (IGF-1) (Diagnostic Products Group). High-sensitive C-reactive protein (Hs-CRP) was measured by immunoturbidimetric assay (Hitachi 917 analyzer), while serum total testosterone was measured by automated 
TABLE 1: Clinical and biochemical characteristics of study participants.

\begin{tabular}{lc}
\hline Age (years) & $69.4 \pm 4.1$ \\
BMI $\left(\mathrm{kg} / \mathrm{m}^{2}\right)$ & $37.0 \pm 4.9$ \\
Weight $(\mathrm{kg})$ & $116.7 \pm 12.9$ \\
Testosterone $(\mathrm{ng} / \mathrm{dL})$ & $119.3 \pm 151.8$ \\
Estradiol $(\mathrm{pg} / \mathrm{mL})$ & $52.5 \pm 22.6$ \\
Adiponectin $(\mathrm{ng} / \mathrm{mL})$ & $25.0 \pm 15.3$ \\
Leptin $(\mu \mathrm{g} / \mathrm{mL})$ & $36.6 \pm 22.6$ \\
IL-6 $(\mathrm{pg} / \mathrm{mL})$ & $2.3 \pm 2.5$ \\
Hs-CRP $(\mathrm{mg} / \mathrm{L})$ & $4.1 \pm 4.5$ \\
sTNFrl $(\mathrm{pg} / \mathrm{mL})$ & $167 \pm 43.4$ \\
PPT $(\mathrm{points})$ & $27.6 \pm 3.3$ \\
Knee flexion $(\mathrm{Nm})$ & $47.7 \pm 16.4$ \\
Knee extension $(\mathrm{Nm})$ & $71.2 \pm 25.5$ \\
Total 1-RM $(\mathrm{lb})$. & $542.7 \pm 194.0$ \\
VO 2 peak $(\mathrm{mL} / \mathrm{kg} / \mathrm{min})$ & $17.2 \pm 3.3$ \\
\hline
\end{tabular}

BMI: Body mass index, IL-6: interleukin-6, Hs-CRP: high sensitivity Creactive protein, sTNFr-1: soluble tumor necrosis factor receptor-1, PPT: physical performance test, Total 1-RM: total 1-repetition max, $\mathrm{VO}_{2}$ peak: peak oxygen consumption, and Nm: Newton meter.

immunoassay (VITROS 5600). The CVs for these assays were $<10 \%$.

2.4. Statistical Analysis. Results are expressed as means \pm SD. A $P$ value of $<0.05$ was considered statistically significant. Normality for outcome variables was verified by ShapiroWilks test. Simple correlation analysis was performed to assess the individual associations between each hormone or adipokine with PPT, knee flexion, knee extension, total 1$\mathrm{RM}$, and $\mathrm{VO}_{2}$ peak followed by multiple regression analysis to determine the independent contribution of each hormone or adipokine to the above tests.

\section{Results}

Our population consisted of 107 frail (PPT of $\leq 32$ ) obese $\left(\mathrm{BMI} \geq 30 \mathrm{~kg} / \mathrm{m}^{2}\right)$ and elderly ( $\geq 65$ years old) males $(n=41)$ and females $(n=66)$. The baseline characteristics of these patients have been reported previously [8] and summarized in Table 1.

Table 2 showed significant positive correlations between the testosterone with the PPT, all measures of strength (knee flexion, knee extension, and total 1-RM), and aerobic fitness $\left(\mathrm{VO}_{2}\right.$ peak), while no correlation was observed between estradiol and any of these measures. Significant positive correlation was observed between IGF-1 and measures of strength and aerobic fitness but not with modified PPT. Among the different adipokines, there was a negative correlation between the proinflammatory cytokine Hs-CRP with PPT, strength, and aerobic fitness $\left(\mathrm{VO}_{2}\right.$ peak). The other pro-inflammatory cytokine IL- 6 also correlated negatively with knee flexion and $\mathrm{VO}_{2}$ peak. The last pro-inflammatory cytokine tested sTNFr1 also correlated negatively with PPT, total 1-RM, and $\mathrm{VO}_{2}$ peak.
The adipokine leptin negatively correlated with PPT, all measures of strength, and $\mathrm{VO}_{2}$ peak. The adipokine adiponectin negatively correlated with total 1-RM.

Multiple regression analysis (Table 3) showed that, for PPT, sTNFrl was the only independent predictor. Independent predictors for knee flexion included adiponectin, leptin and testosterone. Independent predictors for knee extension included: leptin and testosterone. Adiponectin, leptin and testosterone were independent predictors of total 1-RM while independent predictors of peak oxygen consumption $\left(\mathrm{VO}_{2}\right.$ peak) included: IGF-1, IL-6, leptin and testosterone.

\section{Discussion}

Our results demonstrated that although there were correlations between gonadal and anabolic hormones and several adipokines with physical function, different measures of strength, and aerobic fitness $\left(\mathrm{VO}_{2}\right.$ peak), sTNFrl was the only independent predictor of overall physical performance (PPT), while testosterone seemed to be a consistent predictor of all measures of physical strength and fitness. Leptin and adiponectin also appeared to be independent determinants of the different measures of strength, while leptin additionally predicted $\mathrm{VO}_{2}$ peak. On the other hand, IGF-1 only independently predicted physical fitness, while estradiol appeared to have no role in physical function, strength, or fitness. Thus, our results suggest that circulating hormones and adipokines alone or in combination may contribute to frailty and physical disability in obese older adults.

Advancing age is associated with a decline in fat-free mass (FFM; primarily skeletal muscle) and function, known as sarcopenia [14]. Obesity is associated with higher absolute volume of FFM, but there is a disproportionately higher volume of fat mass relative to FFM resulting in relative muscle mass deficit exacerbating age-related muscle loss in the elderly obese [3]. Thus, obesity does not protect against sarcopenia and in fact it acts synergistically with sarcopenia (sarcopenic obesity) resulting in worst disability. Indeed, our group reported that $96 \%$ of obese elderly patients meet criteria for frailty [3].

Both obesity and aging have additive effects on chronic inflammation $[15,16]$ putting the elderly obese in a continuous state of heightened inflammation. Sarcopenic obesity is associated with increased levels of inflammatory cytokines [15] which have direct catabolic effects on skeletal muscle such as: TNF- $\alpha$ which suppresses muscle protein synthesis and interleukin-6 (IL-6) which inhibits the anabolic effects of IGF-1 [7]. Aside from adipose tissue production, skeletal muscles also express cytokines that have direct autocrine and paracrine effects within skeletal muscles [16]. Increased circulating concentrations of IL- 6 are associated with lower muscle mass or strength and impaired mobility and in conjunction with low IGF-1 levels contribute synergistically to produce disability [17]. Our results showed that high levels of proinflammatory cytokines were independently associated with impaired strength and physical function and in fact, sTNFrl was the only independent predictor of physical performance as assessed by the PPT score. 
TABLE 2: Simple correlation analysis between physical function, strength, and aerobic fitness with different hormones and adipokines.

\begin{tabular}{lcccccccc}
\hline & Testosterone & Estradiol & IGF-1 & IL-6 & sTNFr1 & Hs-CRP & Leptin & Adiponectin \\
\hline Modified PPT & $0.23^{*}$ & -0.03 & 0.10 & -0.11 & $-0.30^{* *}$ & $-0.22^{*}$ & $-0.22^{*}$ & -0.01 \\
Knee flexion & $0.49^{* *}$ & 0.14 & $0.26^{*}$ & $-0.25^{*}$ & -0.15 & $-0.22^{*}$ & $-0.53^{* *}$ & -0.11 \\
Knee extension & $0.51^{* *}$ & 0.18 & $0.26^{* *}$ & -0.16 & -0.14 & $-0.26^{* *}$ & $-0.44^{* *}$ & -0.10 \\
Total 1-RM & $0.66^{* *}$ & 0.14 & $0.23^{*}$ & -0.13 & $-0.20^{*}$ & $-0.26^{* *}$ & $-0.52^{* *}$ & $-0.22^{*}$ \\
$\mathrm{VO}_{2}$ peak & $0.56^{* *}$ & 0.04 & $0.20^{*}$ & $-0.28^{* *}$ & $-0.23^{*}$ & $-0.28^{* *}$ & $-0.61^{* *}$ & -0.09 \\
\hline
\end{tabular}

Data are mean $\pm \mathrm{SD} .{ }^{*} P<0.05 ;{ }^{* *} P<0.01 . \mathrm{VO}_{2}$ peak: peak oxygen consumption, Nm: Newton meter, BMI: body mass index, IL-6: interleukin-6, HsCRP: high sensitivity C-reactive protein, sTNFr-1: soluble tumor necrosis factor receptor-1, PPT: modified physical performance test, and Total 1-RM: total 1-repetition max.

TABLE 3: Final model of the hormonal and adipokine predictors of physical performance test, strength measures, and aerobic fitness $\left(\mathrm{VO}_{2}\right.$ peak $)$ in frail obese older adults.

\begin{tabular}{lcccc}
\hline & $R^{2}(\%)$ & Beta estimate & SE & $P$ \\
\hline PPT & 10 & & & \\
$\quad$ sTNFr1 & & -0.025 & 0.008 & 0.003 \\
Knee flexion & 37.9 & & & \\
$\quad$ Adiponectin & & -0.37 & 0.16 & 0.02 \\
$\quad$ Leptin & & -0.29 & 0.09 & 0.002 \\
$\quad$ Testosterone & & 0.034 & 0.013 & 0.01 \\
Knee extension & 27.4 & & & \\
Leptin & & -0.28 & 0.13 & 0.04 \\
$\quad$ Testosterone & & 0.06 & 0.02 & 0.003 \\
Total 1-RM & 54.3 & & & \\
$\quad$ Adiponectin & & -3.22 & 1.07 & 0.004 \\
Leptin & & -2.23 & 0.81 & 0.006 \\
Testosterone & & 0.71 & 0.12 & $<0.001$ \\
VO ${ }_{2}$ peak & 54.3 & & & \\
IGF-1 & & 0.02 & 0.01 & 0.02 \\
IL-6 & & -0.31 & 0.11 & 0.009 \\
Leptin & & -0.05 & 0.01 & $<0.001$ \\
Testosterone & & 0.007 & 0.002 & 0.001 \\
\hline
\end{tabular}

Variables entered in the model: sTNFrl, adiponectin, leptin, testosterone, estradiol, IGF-1, IL-6, leptin, age, and sex. PPT: modified physical performance test, Total 1-RM: total 1-repetition max, $\mathrm{VO}_{2}$ peak: peak oxygen consumption, sTNFrl: soluble tumor necrosis factor receptor-1, IGF-1: insulin growth factor-1, and IL-6: interleukin-6.

Our results also demonstrated a significant contribution of testosterone to physical strength and fitness, while estradiol appeared to have no role in physical function, strength, or fitness in our study population. Although part of decline in physical function and strength with aging is attributed to abnormalities in GH/IGF-1 production and signaling, our study showed that IGF-1 had very little role in these parameters in frail obese older men and women. Aging is associated with decrease in gonadal hormones in both sexes. In women, the drastic drop in estrogen levels with menopause is associated with reduction in muscle mass [18]. In men after age of 40 , testosterone production gradually decreases at a rate of $1.6 \%$ per year for total and to $2-3 \%$ per year for bioavailable testosterone [19]. Because of the age-related increase in sex hormone binding globulin, the magnitude of the decrease in bioavailable testosterone in men is even greater than the decline in total testosterone levels [20]. This reduction in testosterone production in men parallels the (1) age-associated loss of muscle mass that leads to sarcopenia and impairment of function and (2) age-associated loss of bone mass that leads to osteopenia and fracture risk [21, 22].

In men, several randomized controlled trials (RCTs) have demonstrated that testosterone significantly increases FFM (1.1 to $3.7 \mathrm{~kg}$ ) and decreases fat mass (1.1 to $4.5 \mathrm{~kg}$ ) [2325]. In a review of seven trials, testosterone therapy was associated with a significantly greater increase in lean body mass $(2.7 \mathrm{~kg}$; $95 \% \mathrm{CI}, 1.6-3.7)$ and a greater reduction in fat mass (-2.0 kg; 95\% CI 3.1-0.8) than placebo [12]. The body weight change did not differ significantly between groups $(-0.6 \mathrm{~kg} ; 95 \% \mathrm{CI}-2.0-0.8)$. In several studies, the increase in lean mass was accompanied by increase in muscle strength as assessed by hand grip strength, [25] maximum voluntary strength in a leg-press and chest-press exercise, [26] and isokinetic knee extension peak torque [27]. Furthermore, in a meta-analyses of 11 RCTs in elderly men, it was concluded that testosterone increased muscle strength particularly in the lower extremity (effect size: $0.63,95 \% \mathrm{CI}=0.03-1.28$ ) [28]. Moreover, testosterone replacement has been shown to improve physical function in healthy and mostly frail elderly men, as assessed by physical performance test [27], timed physical function test [25], aggregate locomotor function [27], and loaded stair-climbing [26].

On the other hand, although it is well-established that testosterone administration in postmenopausal women resulted in improvement in sexual function not only in surgically menopausal women but also in women with natural menopause [29-31], very few studies have investigated the effects of testosterone administration on body composition, muscle performance, and physical function in women. Huang et al. reported dose-related increases in lean body mass and improvement in strength [32] in postmenopausal women with low gender-adjusted testosterone suggesting the potential for testosterone to improve body composition and physical function in women. Their results were supported by data from another recent study showing that muscle protein synthesis in postmenopausal women is actually stimulated by testosterone and progesterone but not by estradiol [33].

Leptin and adiponectin share similarities in that both are produced by adipose tissues, have receptors in skeletal muscle, and have effects on muscle metabolism [34]. Leptin 
stimulates fatty acid oxidation in skeletal muscles and inhibits fat storage while promoting intramuscular lipolysis $[35,36]$. Nevertheless, despite this positive effect of leptin on muscle metabolism, epidemiologic studies demonstrate a negative association between leptin with muscle mass and function $[13,37]$. This inconsistency is believed to result from leptin resistance that develops with high-fat feeding and obesity $[38,39]$. Similarly for adiponectin, aside from stimulating glucose utilization, it stimulates fatty acid oxidation and inhibits intramuscular fat lipid deposition [40, 41]. However, resistance to adiponectin in peripheral tissues similarly develops with high fat feeding and obesity [42] and may explain the inverse association found between adiponectin, with muscle strength and function $[43,44]$. Thus, these adipokines may be useful as biomarkers for frailty.

Our study has limitations. It is cross-sectional study and therefore does not provide a direct evidence of the actual physiologic role of each factor on physical function, strength, and frailty. In addition, our sample size was relatively small which thus needs confirmation in a larger population of obese older adults.

In summary, our results indicate that circulating testosterone levels and adipokines alone or in combination influence physical function, strength, and aerobic fitness in the frail obese older adults. Our group previously demonstrated that lifestyle intervention by diet and exercise was safe and improves physical function and ameliorates frailty in this population [8]. However, we also showed that although weight loss-associated muscle and bone loss can be attenuated by exercise, it did not totally prevent these complications. Given testosterone's effect on increasing bone density, muscle mass and strength, whether adding testosterone to lifestyle therapy, would be able to prevent muscle and bone loss from weight loss, while at the same time improving strength needs to be examined in future studies.

\section{Conflict of Interests}

The authors declare that there is no conflict of interests regarding the publication of this paper.

\section{Acknowledgment}

This study was supported by Grants RO1-AG025501 and RO1-AG031176 from the National Institutes of Health and resources at the New Mexico VA Health Care System.

\section{References}

[1] G. L. Jensen and P. Y. Hsiao, "Obesity in older adults: relationship to functional limitation," Current Opinion in Clinical Nutrition and Metabolic Care, vol. 13, no. 1, pp. 46-51, 2010.

[2] C. A. Zizza, A. Herring, J. Stevens, and B. M. Popkin, "Obesity affects nursing-care facility admission among whites but not blacks," Obesity Research, vol. 10, no. 8, pp. 816-823, 2002.

[3] D. T. Villareal, M. Banks, C. Siener, D. R. Sinacore, and S. Klein, "Physical frailty and body composition in obese elderly men and women," Obesity Research, vol. 12, no. 6, pp. 913-920, 2004.
[4] S. Basaria, A. D. Coviello, T. G. Travison et al., "Adverse events associated with testosterone administration," The New England Journal of Medicine, vol. 363, no. 2, pp. 109-122, 2010.

[5] T. G. Travison, S. Basaria, T. W. Storer et al., "Clinical meaningfulness of the changes in muscle performance and physical function associated with testosterone administration in older men with mobility limitation," Journals of Gerontology A Biological Sciences and Medical Sciences, vol. 66, no. 10, pp. 1090-1099, 2011.

[6] N. M. Appelman-Dijkstra, K. M. J. A. Claessen, F. Roelfsema, A. M. Pereira, and N. R. Biermasz, "Long-term effects of recombinant human GH replacement in adults with GH deficiency: a systematic review," European Journal of Endocrinology, vol. 169, no. 1, pp. R1-R14, 2013.

[7] F. De Benedetti, T. Alonzi, A. Moretta et al., "Interleukin 6 causes growth impairment in transgenic mice through a decrease in insulin-like growth factor-I. A model for stunted growth in children with chronic inflammation," Journal of Clinical Investigation, vol. 99, no. 4, pp. 643-650, 1997.

[8] D. T. Villareal, S. Chode, N. Parimi et al., "Weight loss, exercise, or both and physical function in obese older adults," The New England Journal of Medicine, vol. 364, no. 13, pp. 1218-1229, 2011.

[9] D. T. Villareal, M. Banks, D. R. Sinacore, C. Siener, and S. Klein, "Effect of weight loss and exercise on frailty in obese older adults," Archives of Internal Medicine, vol. 166, no. 8, pp. 860866, 2006.

[10] D. T. Villareal and J. O. Holloszy, "DHEA enhances effects of weight training on muscle mass and strength in elderly women and men," The American Journal of Physiology-Endocrinology and Metabolism, vol. 291, no. 5, pp. E1003-E1008, 2006.

[11] E. P. Weiss, S. B. Racette, D. T. Villareal et al., "Lower extremity muscle size and strength and aerobic capacity decrease with caloric restriction but not with exercise-induced weight loss," Journal of Applied Physiology, vol. 102, no. 2, pp. 634-640, 2007.

[12] S. Bhasin, G. R. Cunningham, F. J. Hayes et al., “Testosterone therapy in men with androgen deficiency syndromes: an endocrine society clinical practice guideline," Journal of Clinical Endocrinology and Metabolism, vol. 95, no. 6, pp. 2536-2559, 2010.

[13] M. A. Saafi, D. Frere-Meunier, L. Feasson, N. Boutahar, and C. Denis, "Physical fitness is independently related to blood Leptin concentration and insulin sensitivity index in male subjects with central adiposity," Obesity Facts, vol. 5, no. 1, pp. 91-103, 2012.

[14] R. Roubenoff, "Sarcopenia: effects on body composition and function," Journals of Gerontology A Biological Sciences and Medical Sciences, vol. 58, no. 11, pp. 1012-1017, 2003.

[15] M. Cesari, S. B. Kritchevsky, R. N. Baumgartner et al., "Sarcopenia, obesity, and inflammation-results from the Trial of Angiotensin Converting Enzyme Inhibition and Novel Cardiovascular Risk Factors study," The American Journal of Clinical Nutrition, vol. 82, no. 2, pp. 428-434, 2005.

[16] M. Saghizadeh, J. M. Ong, W. T. Garvey, R. R. Henry, and P. A. Kern, "The expression of TNF $\alpha$ by human muscle: Relationship to insulin resistance," Journal of Clinical Investigation, vol. 97, no. 4, pp. 1111-1116, 1996.

[17] A. R. Cappola, Q. L. Xue, L. Ferrucci, J. M. Guralnik, S. Volpato, and L. P. Fried, "Insulin-like growth factor I and interleukin6 contribute synergistically to disability and mortality in older women," Journal of Clinical Endocrinology and Metabolism, vol. 88, no. 5, pp. 2019-2025, 2003. 
[18] V. Messier, R. Rabasa-Lhoret, S. Barbat-Artigas, B. Elisha, A. D. Karelis, and M. Aubertin-Leheudre, "Menopause and sarcopenia: a potential role for sex hormones," Maturitas, vol. 68, no. 4, pp. 331-336, 2011.

[19] H. A. Feldman, C. Longcope, C. A. Derby et al., "Age trends in the level of serum testosterone and other hormones in middleaged men: longitudinal results from the Massachusetts Male Aging Study," Journal of Clinical Endocrinology and Metabolism, vol. 87, no. 2, pp. 589-598, 2002.

[20] S. M. Harman, E. J. Metter, J. D. Tobin, J. Pearson, and M. R. Blackman, "Longitudinal effects of aging on serum total and free testosterone levels in healthy men," Journal of Clinical Endocrinology and Metabolism, vol. 86, no. 2, pp. 724-731, 2001.

[21] S. Khosla, L. J. Melton III, E. J. Atkinson, and W. M. O'Fallon, "Relationship of serum sex steroid levels to longitudinal changes in bone density in young versus elderly men," Journal of Clinical Endocrinology and Metabolism, vol. 86, no. 8, pp. 3555-3561, 2001.

[22] N. Bassil, S. Alkaade, and J. E. Morley, "The benefits and risks of testosterone replacement therapy: a review," Therapeutics and Clinical Risk Management, vol. 5, no. 1, pp. 427-448, 2009.

[23] P. J. Snyder, H. Peachey, P. Hannoush et al., "Effect of testosterone treatment on body composition and muscle strength in men over 65 years of age," Journal of Clinical Endocrinology and Metabolism, vol. 84, no. 8, pp. 2647-2653, 1999.

[24] A. M. Kenny, K. M. Prestwood, C. A. Gruman, K. M. Marcello, and L. G. Raisz, "Effects of transdermal testosterone on bone and muscle in older men with low bioavailable testosterone levels," Journals of Gerontology A Biological Sciences and Medical Sciences, vol. 56, no. 5, pp. M266-M272, 2001.

[25] S. T. Page, J. K. Amory, F. D. Bowman et al., "Exogenous testosterone $(\mathrm{T})$ alone or with finasteride increases physical performance, grip strength, and lean body mass in older men with low serum T,' Journal of Clinical Endocrinology and Metabolism, vol. 90, no. 3, pp. 1502-1510, 2005.

[26] S. Basaria, A. D. Coviello, T. G. Travison et al., "Adverse events associated with testosterone administration," New England Journal of Medicine, vol. 363, no. 2, pp. 109-122, 2010.

[27] U. Srinivas-Shankar, S. A. Roberts, M. J. Connolly et al., "Effects of testosterone on muscle strength, physical function, body composition, and quality of life in intermediate-frail and frail elderly men: a randomized, double-blind, placebo-controlled study," Journal of Clinical Endocrinology and Metabolism, vol. 95, no. 2, pp. 639-650, 2010.

[28] K. J. Ottenbacher, M. E. Ottenbacher, A. J. Ottenbacher, A. A. Acha, and G. V. Ostir, "Androgen treatment and muscle strength in elderly men: a meta-analysis," Journal of the American Geriatrics Society, vol. 54, no. 11, pp. 1666-1673, 2006.

[29] J. L. Shifren, G. D. Braunstein, J. A. Simon et al., “Transdermal testosterone treatment in women with impaired sexual function after oophorectomy," The New England Journal of Medicine, vol. 343, no. 10, pp. 682-688, 2000.

[30] S. R. Davis, M. Moreau, R. Kroll et al., "Testosterone for low libido in postmenopausal women not taking estrogen," The New England Journal of Medicine, vol. 359, no. 19, pp. 2005-2017, 2008.

[31] J. L. Shifren, S. R. Davis, M. Moreau et al., "Testosterone patch for the treatment of hypoactive sexual desire disorder in naturally menopausal women: results from the INTIMATE NM1 Study," Menopause, vol. 13, no. 5, pp. 770-779, 2006.

[32] G. Huang, S. Basaria, T. G. Travison et al., "Testosterone dose-response relationships in hysterectomized women with or without oophorectomy: effects on sexual function, body composition, muscle performance and physical function in a randomized trial," Menopause, vol. 21, no. 6, pp. 612-623, 2014.

[33] G. I. Smith, J. Yoshino, D. N. Reeds et al., "Testosterone and progesterone, but not estradiol, stimulate muscle protein synthesis in postmenopausal women," The Journal of Clinical Endocrinology and Metabolism, vol. 99, no. 1, pp. 256-265, 2014.

[34] D. J. Dyck, "Adipokines as regulators of muscle metabolism and insulin sensitivity," Applied Physiology, Nutrition and Metabolism, vol. 34, no. 3, pp. 396-402, 2009.

[35] G. R. Steinberg, A. Bonen, and D. J. Dyck, "Fatty acid oxidation and triacylglycerol hydrolysis are enhanced after chronic leptin treatment in rats," The American Journal of PhysiologyEndocrinology and Metabolism, vol. 282, no. 3, pp. E593-E600, 2002.

[36] D. M. Muoio, G. Lynis Dohn, F. T. Fiedorek Jr., E. B. Tapscott, and R. A. Coleman, "Leptin directly alters lipid partitioning in skeletal muscle," Diabetes, vol. 46, no. 8, pp. 1360-1363, 1997.

[37] B. Antony, G. Jones, O. Stannus, L. Blizzard, and C. Ding, "Body fat predicts an increase and limb muscle strength predicts a decrease in leptin in older adults over 2. 6 years," Clinical Endocrinology, vol. 79, no. 5, pp. 652-660, 2013.

[38] G. R. Steinberg and D. J. Dyck, "Development of leptin resistance in rat soleus muscle in response to high-fat diets," The American Journal of Physiology-Endocrinology and Metabolism, vol. 279, no. 6, pp. E1374-E1382, 2000.

[39] G. R. Steinberg, M. L. Parolin, G. J. F. Heigenhauser, and D. J. Dyck, "Leptin increases FA oxidation in lean but not obese human skeletal muscle: evidence of peripheral leptin resistance," The American Journal of Physiology - Endocrinology and Metabolism, vol. 283, no. 1, pp. E187-E192, 2002.

[40] T. Yamauchi, J. Kamon, Y. Minokoshi et al., "Adiponectin stimulates glucose utilization and fatty-acid oxidation by activating AMP-activated protein kinase," Nature Medicine, vol. 8, no. 11, pp. 1288-1295, 2002.

[41] E. Tomas, T.-S. Tsao, A. K. Saha et al., "Enhanced muscle fat oxidation and glucose transport by ACRP30 globular domain: Acetyl-CoA carboxylase inhibition and AMP-activated protein kinase activation," Proceedings of the National Academy of Sciences of the United States of America, vol. 99, no. 25, pp. 16309-16313, 2002.

[42] K. L. Mullen, A. C. Smith, K. A. Junkin, and D. J. Dyck, "Globular adiponectin resistance develops independently of impaired insulin-stimulated glucose transport in soleus muscle from high-fat-fed rats," The American Journal of PhysiologyEndocrinology and Metabolism, vol. 293, no. 1, pp. E83-E90, 2007.

[43] G. Loncar, B. Bozic, H. S. von et al., "Association of adiponectin with peripheral muscle status in elderly patients with heart failure," European Journal of Internal Medicine, vol. 24, no. 8, pp. 818-823, 2013.

[44] L. Bucci, S. L. Yani, C. Fabbri et al., "Circulating levels of adipokines and IGF-1 are associated with skeletal muscle strength of young and old healthy subjects," Biogerontology, vol. 14, no. 3, pp. 261-272, 2013. 


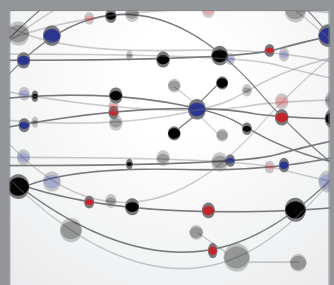

The Scientific World Journal
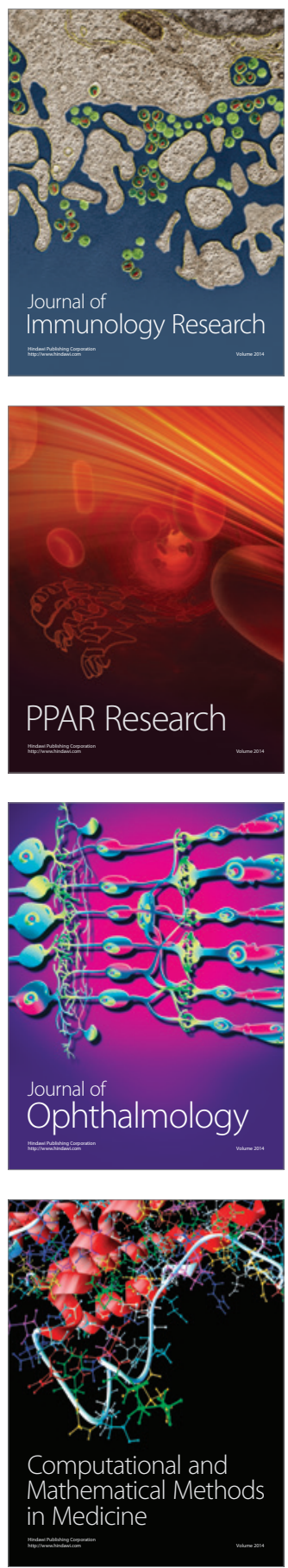

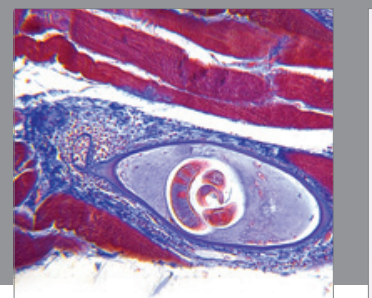

Gastroenterology

Research and Practice
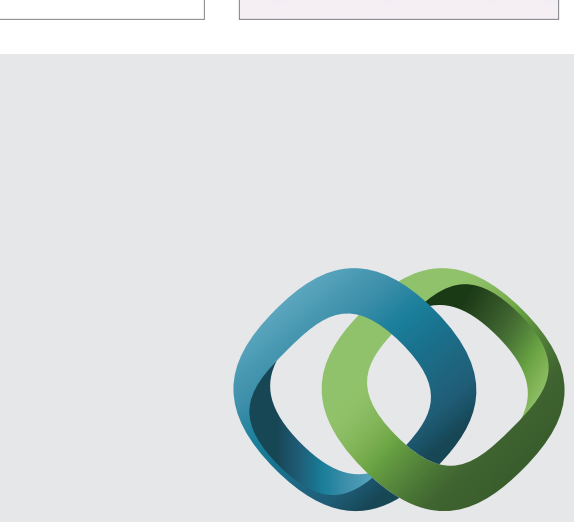

\section{Hindawi}

Submit your manuscripts at

http://www.hindawi.com
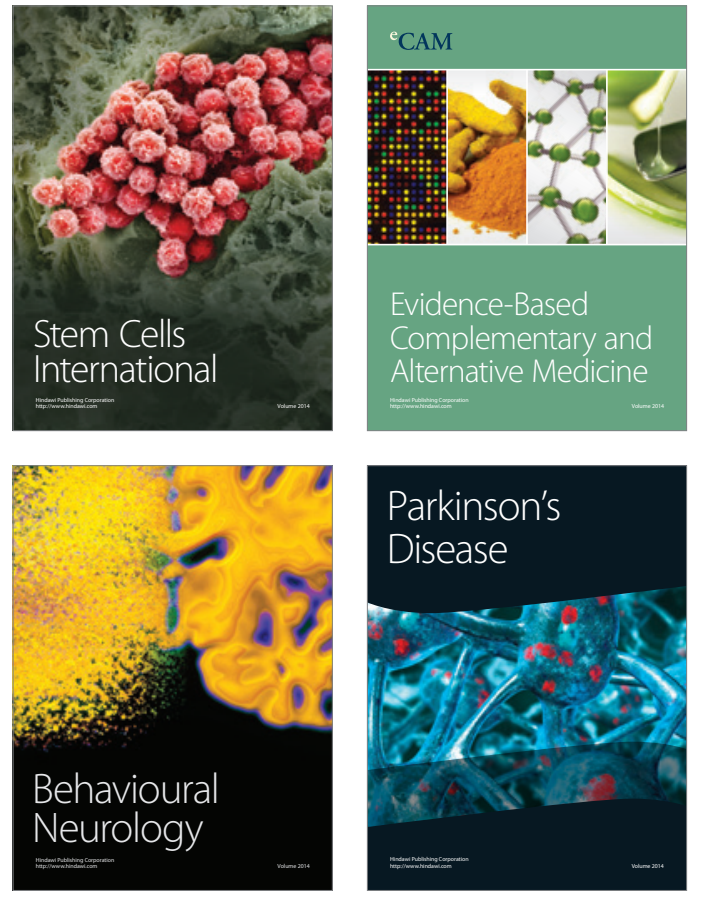
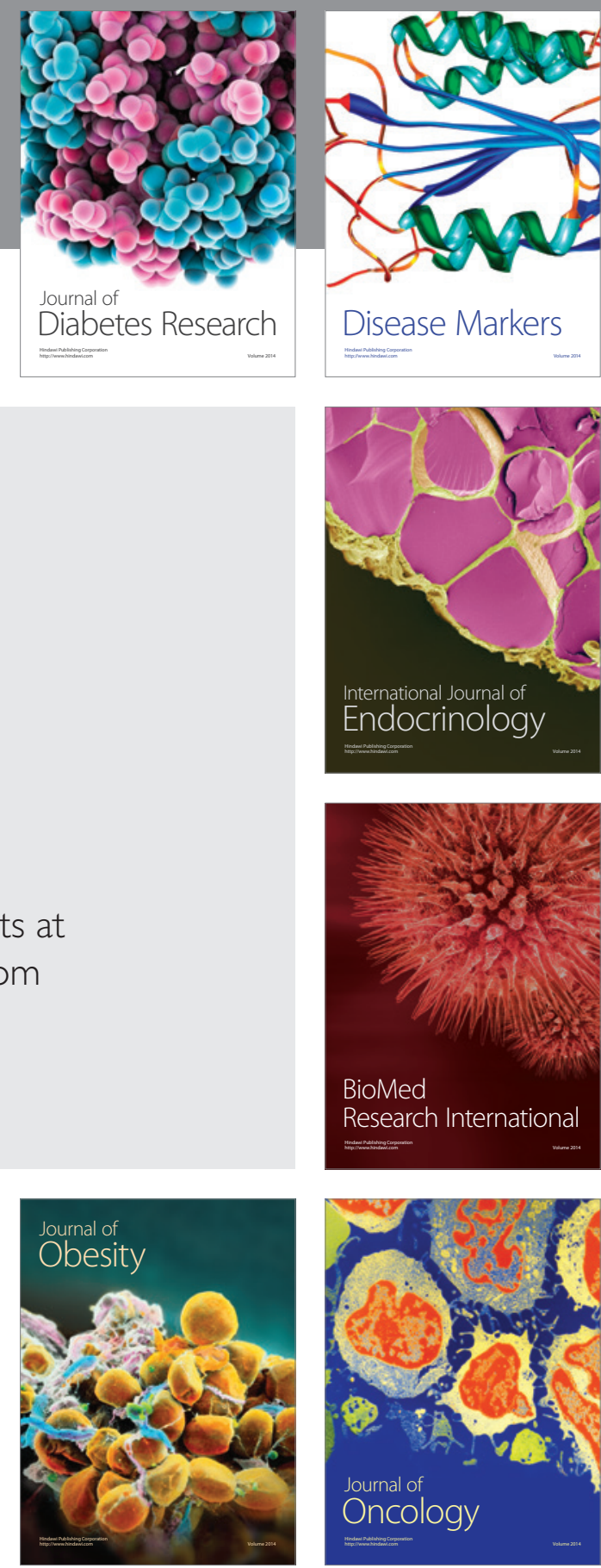

Disease Markers
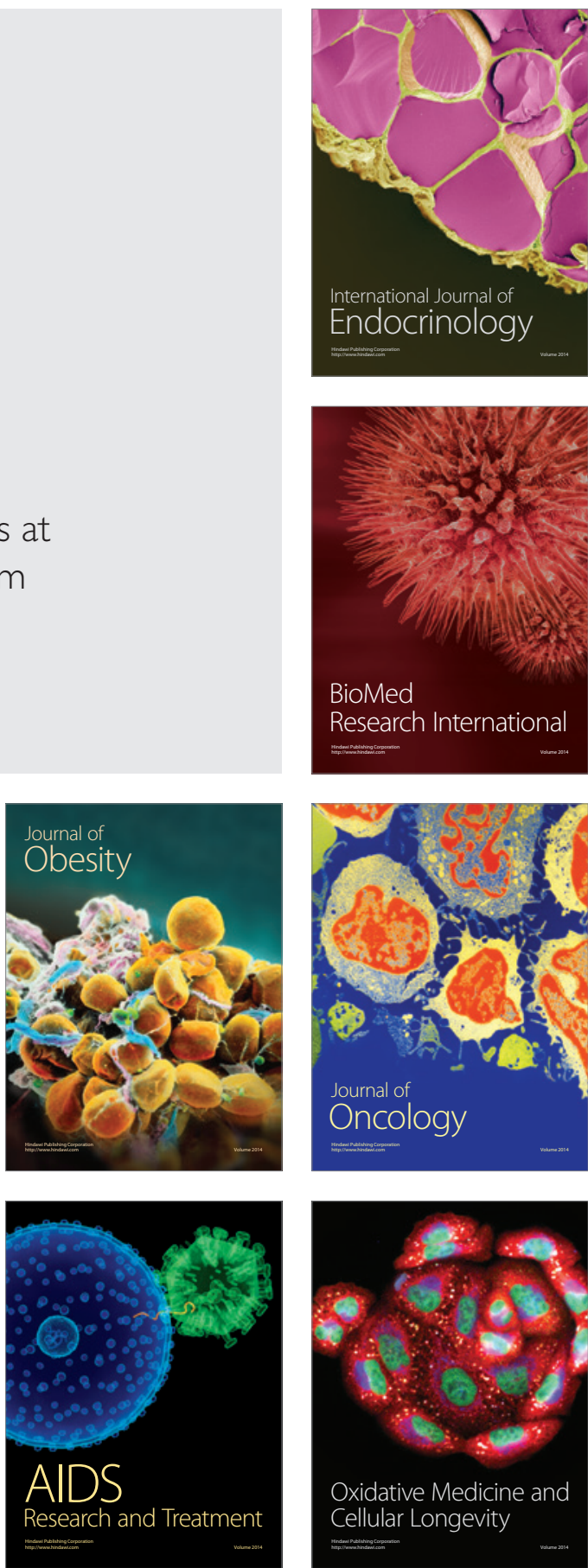